\title{
Inhibitory effect of microRNA-455-5p on biological functions of esophageal squamous cell carcinoma Eca109 cells via Rab31
}

\author{
YING LIU ${ }^{1}$, YANPING TANG ${ }^{1}$ and PING $\mathrm{LI}^{2}$ \\ ${ }^{1}$ Second Department of Gastroenterology, Tianjin Integrated Traditional Chinese and Western Medicine Hospital, \\ Tianjin Nankai Hospital, Tianjin 300100; ${ }^{2}$ College of Acupuncture and Massage, \\ Tianjin University of Traditional Chinese Medicine, Tianjin 300193, P.R. China
}

Received November 3, 2017; Accepted April 14, 2018

DOI: $10.3892 /$ etm.2018.6820

\begin{abstract}
The aim of the present study was to examine microRNA (miRNA or miR)-455-5p expression in esophageal squamous cell carcinoma (ESCC) at the tissue and cellular levels in order to elucidate its biological roles. A total of 60 patients with ESCC were enrolled in the present study and reverse transcription-quantitative polymerase chain reaction was used to measure the expression of miR-455-5p. ESCC Eca109 cells were transfected with miR-NC, miR-455-5p mimics or inhibitor and a Cell Counting Kit-8 assay was used to assess proliferation. To investigate the migration and invasion abilities of Eca109 cells, Transwell and Matrigel assays were performed. Western blotting was employed to measure Rab31 protein expression, while a rescue assay was utilized to study the biological roles of miR-455-5p and Rab31 in Eca109 cells. To determine whether Rab31 is a direct target of miR-455-5p, a dual luciferase reporter assay was performed. The results revealed that miR-455-5p expression was decreased in ESCC tissues and was negatively correlated with metastasis and pathogenesis. In vitro overexpression of miR-455-5p inhibited the proliferation, migration and invasion of ESCC Eca109 cells. Furthermore, miR-455-5p regulated the expression of Rab31 protein in Eca109 cells. Rab31 overexpression promoted the proliferation, migration and invasion of Eca109 cells. Luciferase reporter assay results revealed that miR-455-5p is able to bind with the 3'-untranslated region of Rab31 mRNA to regulate its expression. In summary, the results of the present study suggest that miR-455-5p expression is decreased in ESCC tissues and is miR-455-5p is negatively correlated with lymphatic metastasis and differentiation. As a tumor-suppressor gene, miR-455-5p inhibits the proliferation,
\end{abstract}

Correspondence to: Dr Yanping Tang, Second Department of Gastroenterology, Tianjin Integrated Traditional Chinese and Western Medicine Hospital, Tianjin Nankai Hospital, 122 Sanwei Road, Tianjin 300100, P.R. China

E-mail: tangyp17@163.com

Key words: microRNA-455-5p, esophageal squamous cell carcinoma, Eca109 cells, Rab31 migration and invasion of ESCC Eca109 cells by suppressing the expression of Rab31.

\section{Introduction}

Esophageal carcinoma is a common malignant tumor of the digestive tract, mainly comprising squamous cell carcinoma and adenocarcinoma, that is typically associated with poor patient prognosis $(1,2)$. In China, esophageal carcinoma is has a high incidence and is the fourth most common of all malignant tumors (3). In addition, 90\% cases of esophageal carcinoma are squamous cell carcinoma (4). Although clinical diagnostic methods and treatments for esophageal squamous cell carcinoma (ESCC) have improved in recent years, the 5-year survival rate of patients with ESCC remains 20\% $(5,6)$. Distant metastasis is the major cause of poor patient prognosis (7). It has been reported that the invasion and metastasis of tumor cells is a complex process comprising multiple genes, steps and stages, and the molecular mechanism that regulates this process is not clear (8). It is therefore important to understand the molecular mechanisms underlying the invasion and metastasis of ESCC and to identify novel genes involved.

MicroRNA (miRNA or miR) is a class of non-coding small RNA molecules (18-22 nucleotides) that regulate gene expression at the post-transcriptional level (9). miRNAs are associated with the regulation of tumor cell proliferation, invasion, metastasis, apoptosis and autophagy, as well as being important molecular targets for early diagnosis and treatment of cancer (10). It has been reported that the expression of a number of miRNAs in ESCC tissues and the peripheral blood is disordered; this may be of great value in clinical diagnosis and prognosis (11). For example, miR-506 expression is significantly elevated in the peripheral blood of patients with ESCC and is positively correlated with lymphatic metastasis, TNM staging and tumor size (12). Furthermore, the expression of miR-1297 in the peripheral blood of patients with ESCC is significantly reduced and can be used as a diagnostic marker (13). miR-613 is significantly downregulated in ESCC tissues and the peripheral blood, as well as being negatively correlated with lymphatic metastasis and TNM staging; Kaplan-Meier analysis has been used to demonstrate that low miR-613 expression is associated with poor patient prognosis (14). It has also been reported that miR-10b, miR-29c and 
miR-205 have good specificity and sensitivity as predictors of diagnosis or prognosis in ESCC (15). Together, these studies suggest that miRNAs may be valuable in the early diagnosis, clinical treatment and prognosis of ESCC.

miR-455 is a tumor-associated miRNA molecule whose gene is located at fragile site region on chromosome $9 \mathrm{q} 32(16,17)$. miR-455 is abnormally expressed in a number of tumors, including breast cancer, non-small cell lung cancer and gastric cancer (18). Mature miR-455 includes two subtypes; miR-455-3p and miR-455-5p (19). miR-455-3p is associated with tumor proliferation, invasion and metastasis; for example, miR-455-3p inhibits proliferation and induces apoptosis in colon cancer HCT116 cells (20). miR-455-3p promotes the invasion and metastasis of triple negative breast cancer cells by targeting EI24 (21). By contrast, there have been few studies into the effect of miR-455-5p in tumors and it is thought that its biological function is associated with the tumor tissue type (22). For example, miR-455-5p inhibits the proliferation and metastasis of gastric cancer by downregulating the expression of Rab18, acting as a tumor-suppressor gene (23). In oral squamous cell carcinoma, the transforming growth factor (TGF)- $\beta$-SMAD signaling pathway upregulates miR-455-5p expression and promotes tumor cell proliferation by targeting UBE2B (24). However, the expression and mechanism of action of miR-455-5p in ESCC remain unclear. Rab31 is a tumor-associated gene that has been reported in recent years (25). The expression of Rab31 is abnormal in a variety of tumor types and Rab31 serves a role in the proliferation, invasion and metastasis of tumor cells $(26,27)$. Furthermore, the association between Rab31 and miR-455-5p in ESCC has not previously been reported, nor has the role of Rab31. The aim of the present study was to investigate the expression and underlying mechanisms of miR-455-5p and Rab31 in ESCC at the cellular and molecular levels.

\section{Materials and methods}

Patients. A total of 60 patients (36 males and 24 females) with ESCC who received radical or palliative resection of tumor tissues (ESCC group) and tumor-adjacent tissues (control group) at Tianjin Integrated Traditional Chinese and Western Medicine Hospital (Tianjin, China) between December 2014 and January 2016 were included in the present study. ESCC was diagnosed and classified by two pathologists according to the 2003 WHO cancer classification criteria (28). The resected tissues were stored at $-80^{\circ} \mathrm{C}$ before use. The age range of the patients was $36-73$ years and the mean age was $51 \pm 1.8$ years. None of the patients received adjuvant therapy prior to the surgery. Patients with lymph node metastasis ( 29 cases) were included in the N1 subgroup, while those without lymph node metastasis (31 cases) were included in the N0 subgroup. According to the classification of differentiation degrees (29), 11 patients had poor differentiation, 28 patients had moderate differentiation and 21 patients had high differentiation. All procedures were approved by the Ethics Committee of Tianjin Nankai Hospital (Tianjin, China). Written informed consent was obtained from all patients or their families.

Cells. The ESCC cell line Eca109 was purchased from the Shanghai Institute of Cell Biology (Chinese Academy of
Sciences, Shanghai, China). Cells were cultured in fresh RPMI-1640 medium supplemented with $10 \%$ fetal bovine serum (FBS) (both Thermo Fisher Scientific, Inc., Waltham, $\mathrm{MA}, \mathrm{USA}$ ) at $37^{\circ} \mathrm{C}$ in an atmosphere containing $5 \% \mathrm{CO}_{2}$. The medium was replaced every two days and cells were passaged when they reached $80-90 \%$ confluence.

Eca109 cells were divided into the following groups: Negative control (NC), miR-455-5p mimics (5'-TAUGTG CCTTTGGACTACATCG-3') and miR-455-5p inhibitor (5'-GCAGTCCATGGGCATATACAC-3'). When cells reached $70-90 \%$ confluence, $1.25 \mu \mathrm{lmiR}-455-5 \mathrm{p}$ mimics or miR-455-5p inhibitor (20 pmol $/ \mu \mathrm{l}$; Gangzhou RiboBio Co., Ltd., Guangzhou, China) and $2 \mu 1$ Lipofectamine ${ }^{\circledR} 2000$ (Thermo Fisher Scientific, Inc.) were added into two individual vials containing $50 \mu \mathrm{l}$ Opti Mem medium (Thermo Fisher Scientific, Inc.), respectively, for $5 \mathrm{~min}$. The contents of the vials were mixed together and left to stand for $15 \mathrm{~min}$. The mixture was subsequently applied to the cells and incubated at $37^{\circ} \mathrm{C}$ for $6 \mathrm{~h}$, following which the medium was replaced with RPMI-1640 supplemented with $10 \%$ FBS. Cells were cultured at $37^{\circ} \mathrm{C}$ in an atmosphere containing $5 \% \mathrm{CO}_{2}$ for $48 \mathrm{~h}$ prior to use.

For rescue experiments, Eca109 cells $\left(2 \times 10^{5}\right)$ in the miR-NC, miR-455-5p mimics and inhibitor groups were seeded into 24 -well plates ( $1 \times 10^{5} /$ well) containing antibiotic-free RPMI-1640 medium supplemented with 10\% FBS. When cells reached $60 \%$ confluence, Eca109 cells in the miR-455-5p mimics and inhibitor groups were transfected with $0.5 \mu \mathrm{g}$ pcDNA-3.1-Rab31 or pcDNA-3.1-shR-Rab31 plasmids (Hanbio Biotechnology Co., Ltd., Shanghai, China) using Lipofectamine 2000 (Thermo Fisher Scientific, Inc.), respectively, while cells in the miR-NC group were transfected with $0.5 \mu \mathrm{g}$ negative control (NC) plasmid. Cells were cultured at $37^{\circ} \mathrm{C}$ in an atmosphere containing $5 \% \mathrm{CO}_{2}$ for $6 \mathrm{~h}$, following which the medium was replaced with fresh RPMI-1640 containing 10\% FBS and cultured for $48 \mathrm{~h}$.

Reverse transcription-quantitative polymerase chain reaction ( $R T-q P C R)$. ESCC and tumor-adjacent tissues $(100 \mathrm{mg})$ were ground into powder using liquid nitrogen and mixed with $1 \mathrm{ml}$ TRIzol (Thermo Fisher Scientific, Inc.) for lysis. Total RNA was subsequently extracted using the phenol chloroform method. The purity of RNA was determined using A260/A280 ultraviolet spectrophotometry (Nanodrop ND2000; Thermo Fisher Scientific, Inc., Pittsburgh, PA, USA). cDNA was obtained by RT using a PrimeScript RT Reagent Kit (Takara Bio, Inc., Otsu, Japan) and stored at $-20^{\circ} \mathrm{C}$. The qPCR reaction system comprised $10 \mu \mathrm{l}$ qRT-PCR-Mix (miScript SYBR Green PCR Kit; Qiagen GmbH, Hilden, Germany), $0.5 \mu 1$ upstream primer (5'-TATGTGCCTTTG GACTACATCG-3'), $0.5 \mu 1$ downstream primer (universal primer provided with kit; cat. no. 218073; Qiagen $\mathrm{GmbH}$ ),

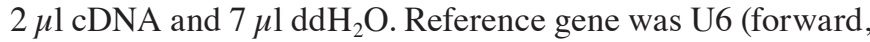
5'-GCGCGTCGTGAAGCGTTC-3'; reverse, 5'-GTGCAG GGTCCGAGGT-3'). Thermocycling condition were as follows: Initial denaturation at $95^{\circ} \mathrm{C}$ for $10 \mathrm{~min}$, followed by 40 cycles of denaturation at $95^{\circ} \mathrm{C}$ for $1 \mathrm{~min}$ and elongation at $60^{\circ} \mathrm{C}$ for $30 \mathrm{sec}$. The $2^{-\Delta \Delta \mathrm{Cq}}$ method (30) was used to calculate relative expression of target mRNA against U6. Each sample was tested in triplicate. 
Cell Counting Kit (CCK)-8 assay. Sample cells were inoculated in 96 -well plates at a density of 2,000 cells/well. At $0,24,48$ and $72 \mathrm{~h}, 20 \mu \mathrm{l} \mathrm{CCK}-8$ reagent $(5 \mathrm{~g} / \mathrm{l}$; Beyotime Institute of Biotechnology, Haimen, China) was added at $37^{\circ} \mathrm{C}$ for $2 \mathrm{~h}$. The absorbance $(490 \mathrm{~nm})$ of each well was measured and cell proliferation curves were plotted. Each group was tested in 3 replicate wells.

Transwell assay. Transwell chambers $(8 \mu \mathrm{m}$ diameter and 24 wells; Corning Inc., Corning, NY, USA) were used to evaluate the migration of Eca109 cells. Transfected cells were collected by trypsin digestion and resuspended at a density of $2 \times 10^{5}$ cells/ml in RPMI-1640 medium. The cell suspension $(200 \mu \mathrm{l})$ was added into the upper chamber. A total of $500 \mu \mathrm{l}$ RPMI-1640 medium supplemented with 10\% FBS was added to the lower chamber. Following $24 \mathrm{~h}$ of incubation at $37^{\circ} \mathrm{C}$, cells in the upper chamber were removed using a cotton swab. The chamber was subsequently fixed using $4 \%$ formaldehyde for $10 \mathrm{~min}$ at room temperature, subjected to Giemsa's staining at room temperature for $1 \mathrm{~min}$ and washed three times. Cells that had migrated to the lower chamber were counted under a microscope (5 fields; magnification, x200) to evaluate migration ability.

Matrigel invasion chambers (BD Biosciences, Franklin Lakes, NJ, USA) were used to determine the invasion ability of cells. Matrigel (BD Biosciences, Franklin Lakes, NJ, USA) was first diluted with serum-free RPMI-1640 medium at a ratio of $1: 2$. In the upper chamber, $100 \mu 1$ diluted Matrigel was added and incubated at $37^{\circ} \mathrm{C}$ for $1 \mathrm{~h}$. A total of $500 \mu \mathrm{l}$ RPMI-1640 medium supplemented with $10 \%$ FBS was added to the lower chamber. Following incubation at $37^{\circ} \mathrm{C}$ for $72 \mathrm{~h}$, cells in the upper chamber were removed using a cotton swab. The chamber was subsequently fixed using $4 \%$ formaldehyde for $10 \mathrm{~min}$ at room temperature and then subjected to Giemsa's staining at room temperature for $1 \mathrm{~min}$. Following three washes, 3 cells which had invaded the lower chamber were counted under a microscope (5 fields; magnification, x200).

Western blotting. Cells in each group were trypsinized and collected. Cold radioimmunoprecipitation assay lysis buffer (600 $\mu \mathrm{l}$; Beyotime Institute of Biotechnology) was mixed with the samples for $30 \mathrm{~min}$ on ice, following which samples were centrifuged at $10,000 \times \mathrm{g}$ and $4^{\circ} \mathrm{C}$ for $10 \mathrm{~min}$. The protein concentration was determined using a bicinchoninic acid protein concentration determination kit (RTP7102; Realtimes Biotechnology Co., Ltd., Beijing, China). Protein samples $(5 \mu \mathrm{l})$ were separated by $10 \%$ SDS-PAGE and electro-transferred to polyvinylidene difluoride membranes on ice. Membranes were blocked with 5\% skimmed milk at room temperature for $1 \mathrm{~h}$. Subsequently, membranes were incubated with rabbit anti-human Rab31 polyclonal primary antibody (1:1,000; BS70807) and rabbit anti-human GAPDH primary antibody (1:5,000; AP0063; both Bioworld Technology, Inc., St. Louis Park, MN, USA) at $4^{\circ} \mathrm{C}$ overnight. Membranes were washed with PBS with Tween 20 (PBST) and incubated with goat anti-mouse horseradish peroxidase-conjugated secondary antibodies (1:2,000; BS12478; Bioworld Technology, Inc.) at room temperature for $1 \mathrm{~h}$. Membranes were washed five times with PBST for 5 min and developed using an enhanced chemiluminescence detection kit (Sigma-Aldrich; Merck KGaA,
Darmstadt, Germany) for imaging. Image lab v3.0 software (Bio-Rad Laboratories, Inc., Hercules, CA, USA) was used to acquire and analyze imaging data. The relative expression of Rab31 protein was calculated with GAPDH as a control.

Dual luciferase reporter assay. To investigate the regulatory mechanism of Rab31, TargetScan (http://www.targetscan.org) was used to predict miRNA molecules that might regulate Rab31. It was demonstrated that miR-455-5p was a potential regulator of Rab31 expression. Wild-type (WT) and mutant seeding regions of miR-455-5p in the 3'-UTR of Rab31 were chemically synthesized, following which SpeI and HindIII restriction sites were added and cloned into pMIR-REPORT luciferase reporter plasmids $(0.5 \mu \mathrm{g}$; Thermo Fisher Scientific, Inc.) with WT or mutant 3'-UTR DNA sequences. Plasmids were transfected together with miR-NC or miR-455-5p mimics into Eca109 cells as described earlier. Following incubation for $24 \mathrm{~h}$, cells were processed using dual luciferase reporter assay kit according to the manufacturer's protocol (Promega Corp., Madison, WI, USA). The fluorescence intensity was measured using a GloMax 20/20 luminometer (Promega Corp.). Fluorescence values of each group of were measured using Renilla fluorescence activity as the internal reference.

Statistical analysis. All results were analyzed using SPSS 17.0 statistical software (SPSS, Inc., Chicago, IL, USA). Student's t test was used for comparisons between two groups. Multiple group comparisons were analyzed using one-way analysis of variance with least significant difference and Student-Newman-Keul's post hoc tests. For heterogeneous data, Tamhane's T2 or Dunnett's T3 post hoc tests were used. $\mathrm{P}<0.05$ was considered to indicate a statistically significant difference.

\section{Results}

miR-455-5p expression is decreased in ESCC tissues and is negatively correlated with metastasis and development of the disease. To measure miR-455-5p expression in ESCC tissues, RT-qPCR was performed. The results demonstrated that miR-455-5p expression was significantly decreased in ESCC tissues compared with tumor-adjacent tissues $(\mathrm{P}<0.01$; Fig. 1A). In addition, miR-455-5p expression in ESCC tissues from patients with lymphatic metastasis was significantly reduced compared with tissues from patients without lymphatic metastasis ( $\mathrm{P}<0.01$; Fig. 1B). miR-455-5p was also significantly downregulated in ESCC tissues from patients with poor differentiation compared with tissues from patients with high or moderate differentiation $(\mathrm{P}<0.05$; Fig. 1C). These results suggest that miR-455-5p expression is decreased in ESCC tissues and is negatively correlated with metastasis and disease development.

miR-455-5p inhibits the proliferation of Ecal09 cells. A CCK-8 assay was performed to assess proliferation. The results revealed that the absorbance of Eca109 cells t transfected with miR-455-5p mimics was significantly reduced compared with the miR-NC group at all time points $(\mathrm{P}<0.05)$, while the absorbance of Eca109 cells in the miR-455-5p inhibitor group was significantly increased compared with the miR-NC 

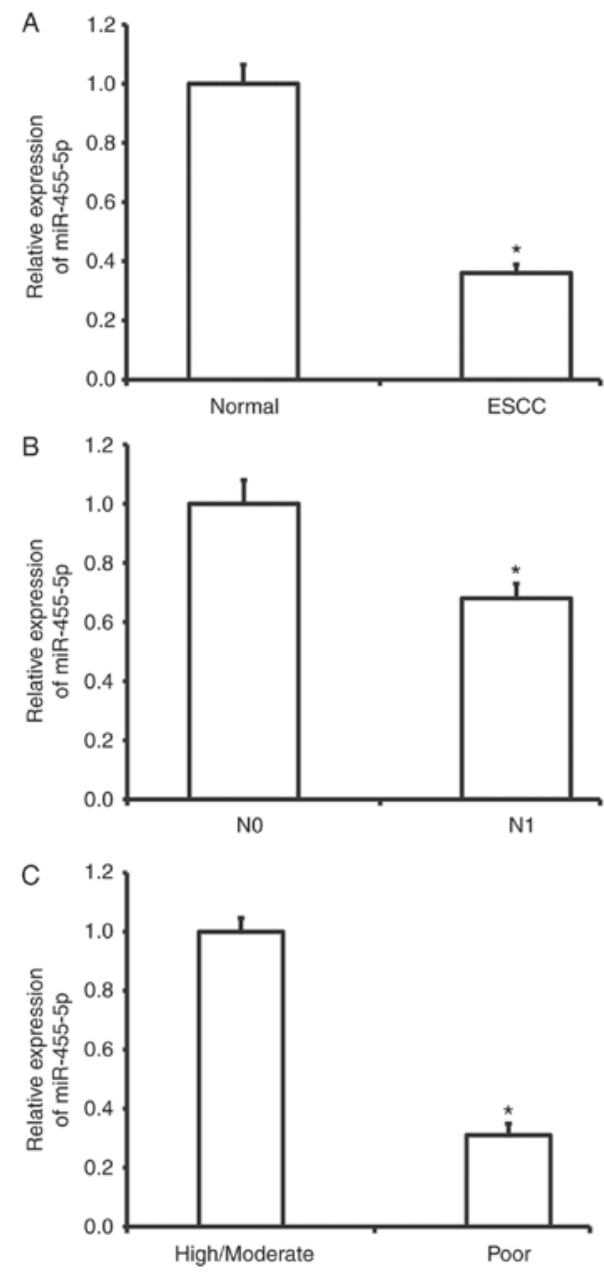

Figure 1. Expression of miR-455-5p in ESCC tissues. (A) Expression of miR-455-5p in tumor-adjacent normal tissues and ESCC tissues. "P<0.05 vs. normal group. (B) Expression of miR-455-5p in ESCC tissues from patients with ESCC with (N1) or without (N0) lymphatic metastasis. ${ }^{*} \mathrm{P}<0.05$ vs. N0 group. (C) Expression of miR-455-5p in ESCC tissues from patients with different amounts of differentiation of the disease. ${ }^{\text {}} \mathrm{P}<0.05$ vs. patients with high and moderate degrees of ESCC. miR, microRNA; ESCC, esophageal squamous cell carcinoma.

group ( $\mathrm{P}<0.05$; Fig. 2). These results indicate that miR-455-5p expression inhibits ESCC Eca109 cell proliferation.

miR-455-5p expression inhibits the migration and invasion abilities of Ecal09 cells. A Transwell assay was used to investigate the migration and invasion abilities of Eca109 cells. The results revealed fewer cells in crossed the chamber membrane the miR-455-5p mimics group compared with the miR-NC group $(\mathrm{P}<0.05$; Fig. 3). In contrast, the number of cells in the miR-455-5p inhibitor group that crossed the chamber membrane was significantly increased compared with the miR-NC group $(\mathrm{P}<0.05$; Fig. 3$)$. In the invasion assay, the number of cells that crossed the chamber membrane was lower in the miR-455-5p mimics group compared with the miR-NC group $(\mathrm{P}<0.05$; Fig. 3$)$. In the miR-455-5p inhibitor group, there was a significant increase in the number of invading cells compared with the miR-NC group $(\mathrm{P}<0.05$; Fig. 3). These results suggest that miR-455-5p expression inhibits the migration and invasion abilities of Eca109 cells.

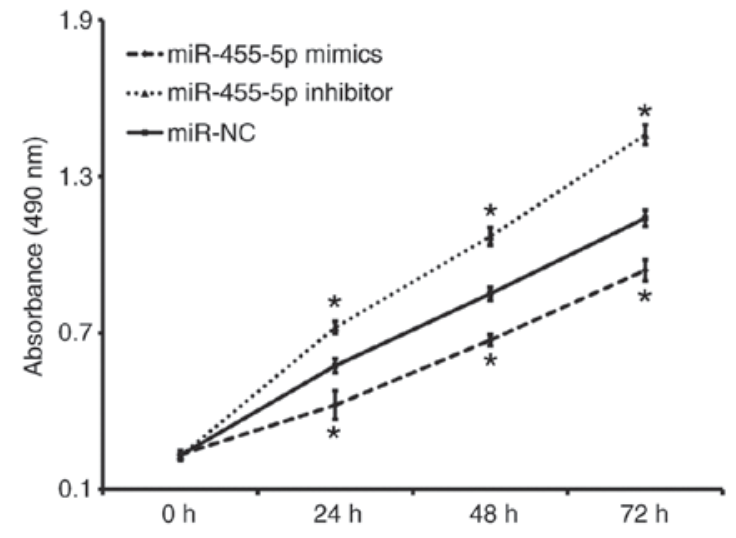

Figure 2. Effect of miR-455-5p on the proliferation of ESCC Eca109 cells transfected with miR-NC, miR-455-5p mimics or miR-455-5p inhibitor as measured using a Cell Counting Kit- 8 assay. ${ }^{*} \mathrm{P}<0.05$ vs. miR-NC group. miR, microRNA; ESCC, esophageal squamous cell carcinoma; NC, negative control.

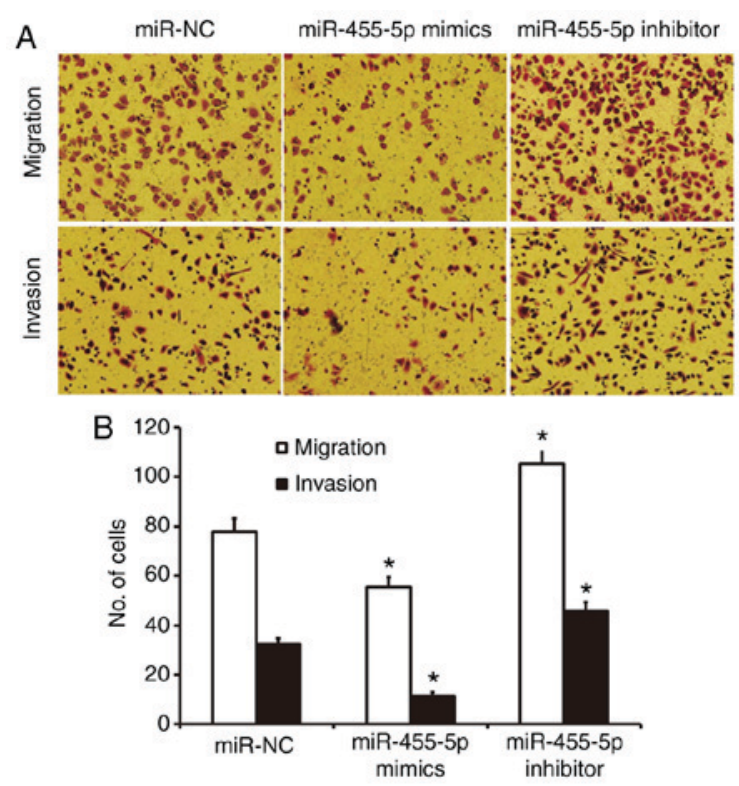

Figure 3. Effect of miR-455-5p on the migration and invasion of ESCC Eca109 cells. Cells were transfected with miR-NC, miR-455-5p mimics or miR-455-5p inhibitor. (A) Images of and (B) quantified data for migrated and invaded cells in each group. ${ }^{*} \mathrm{P}<0.05$ vs. miR-NC group. miR, microRNA; ESCC, esophageal squamous cell carcinoma; $\mathrm{NC}$, negative control.

Rab31 expression is regulated by miR-455-5p in Eca109 cells. Western blotting was performed to assess Rab31 protein expression in Eca109 cells. The results revealed that Rab31 protein expression was significantly reduced in the miR-455-5p mimics group compared with the miR-NC group $(\mathrm{P}<0.05)$, while it was significantly increased in the miR-455-5p inhibitor group ( $\mathrm{P}<0.05$; Fig. 4). These results suggest that miR-455-5p regulates the expression of Rab31 protein in Eca109 cells.

Rab31 promotes the proliferation, migration and invasion of ESCC Eca109 cells. To assess how the regulation of Rab31 by miR-455-5p affects the biological functions of Eca109 cells, Rab31 protein was silenced or overexpressed. The results of a CCK-8 assay revealed that miR-455-5p-induced Rab31 


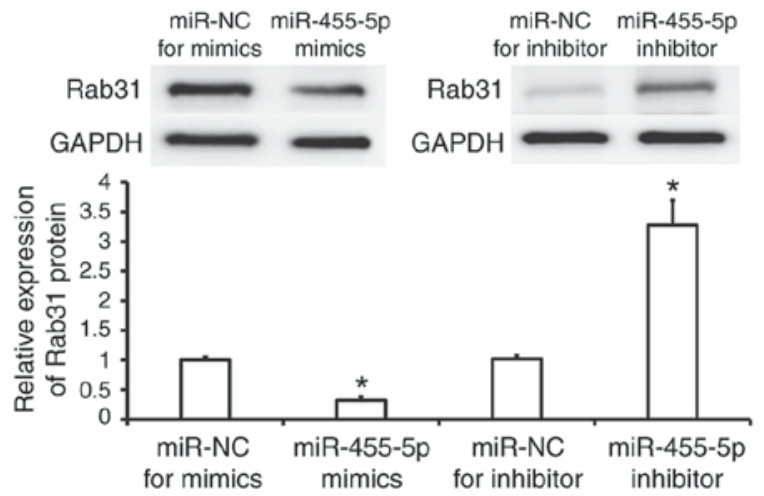

Figure 4. Expression of Rab31 protein in ESCC Eca109 cells. Cells were transfected with miR-NC, miR-455-5p mimics, miR-NC or miR-455-5p inhibitor. Rab31 protein was measured using Western blotting. ${ }^{*} \mathrm{P}<0.05$ vs. miR-NC groups. miR, microRNA; ESCC, esophageal squamous cell carcinoma; NC, negative control.

downregulation significantly decreased the proliferation of Eca109 cells $(\mathrm{P}<0.05$; Fig. 5A). However, co-transfection with pcDNA3.1-Rab31 (which reversed Rab31 downregulation) increased the proliferation of Eca109 cells to a level higher than the miR-NC group ( $\mathrm{P}<0.05$; Fig. $5 \mathrm{~A})$. Rab31 overexpression induced by the inhibition of miR-455-5p significantly promoted the proliferation of Eca109 cells $(\mathrm{P}<0.05)$, whereas Rab31 downregulation induced by co-transfection with pcDNA3.1-shR-Rab31 reduced the proliferation of Eca109 cells to a level similar to that observed in the miR-NC group (Fig. 5B). Transwell assay data revealed that cotransfection with pcDNA3.1-Rab31 increased the migration and invasion abilities of Eca109 cells to levels similar to those observed in the miR-NC group ( $\mathrm{P}<0.05$; Fig. 5C), whereas Rab31 downregulation induced by miR-455-5p overexpression reduced migration and invasion compared with the miR-NC group $(\mathrm{P}<0.05$; Fig. 5D). Rab31 overexpression induced by miR-455-5p inhibition resulted in increased migration and invasion compared with the miR-NC group $(\mathrm{P}<0.05)$, whereas co-transfection with pcDNA3.1-shR-Rab31 (which downregulated Rab31 expression) reduced the migration and invasion abilities of Eca109 cells to levels similar to the miR-NC group (Fig. 5D). The results indicate that Rab31 promotes the proliferation, migration and invasion of ESCC Eca109 cells.

miR-455-5p binds with the 3'-UTR seeding region of Rab31 $m R N A$ to regulate its expression. To investigate the interaction between miR-455-5p and the 3'-UTR of Rab31 mRNA, a dual luciferase reporter assay was performed. The fluorescence value of cells co-transfected with miR-455-5p mimics and pMIR-REPORT-WT luciferase reporter plasmids was significantly lower compared with the NC group $(\mathrm{P}<0.05$; Fig. 6). In contrast, no significant difference was observed in the fluorescence of cells co-transfected with miR-455-5p mimics and pMIR-REPORT-mutant luciferase reporter plasmids compared with the NC group (Fig. 6). These results indicate that miR-455-5p is able to bind with the 3'-UTR seeding region of Rab31 mRNA to regulate its expression.

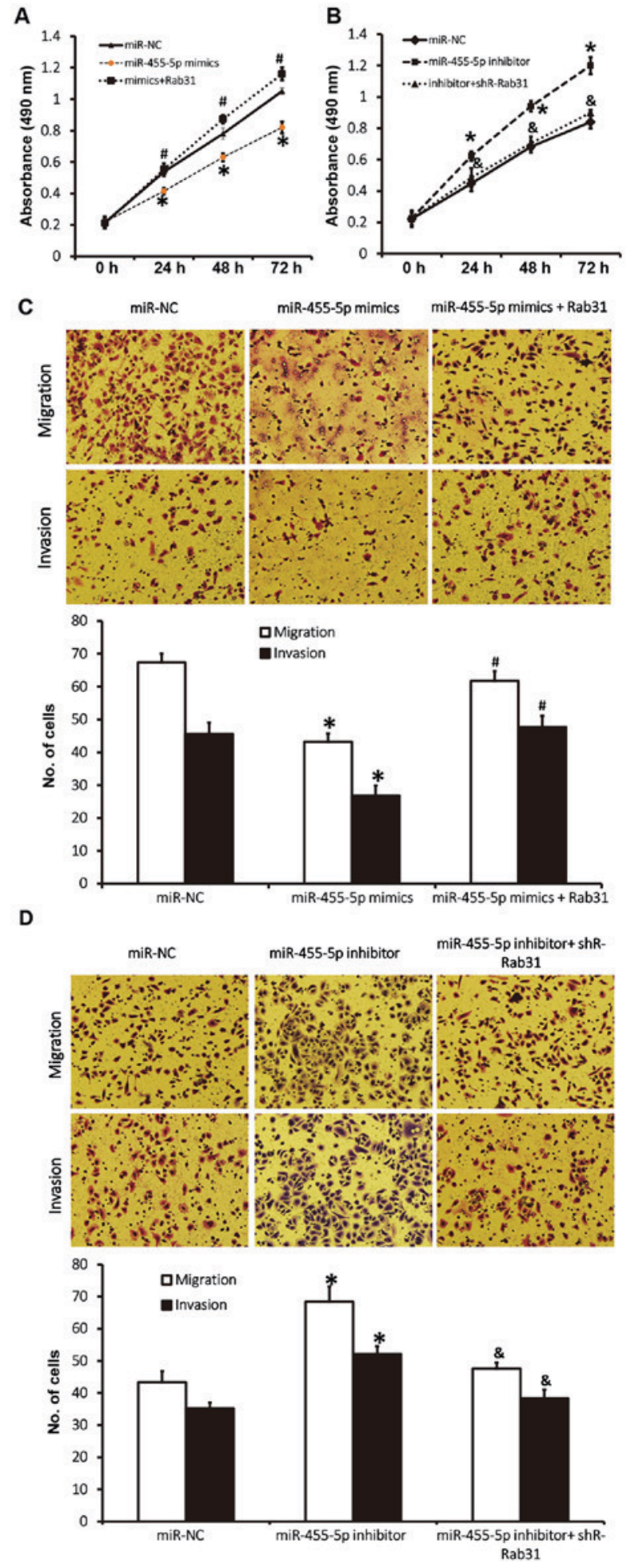

Figure 5. Effect of Rab31 expression on proliferation, migration and invasion in ESCC Eca109 cells. (A) Proliferation of Eca109 cells following transfection with miR-NC, miR-455-5p mimics or miR-455-5p mimics + Rab31. " $\mathrm{P}<0.05$ vs. miR-NC group and ${ }^{\#} \mathrm{P}<0.05$ vs. miR- $455-5 \mathrm{p}$ mimics group. (B) Proliferation of Eca109 cells following transfection with miR-NC, miR-455-5p inhibitor or miR-455-5p inhibitor + shR-Rab31. ${ }^{*} \mathrm{P}<0.05$ vs. miR-NC group and ${ }^{\circledR} \mathrm{P}<0.05$ vs. miR-455-5p inhibitor group. Cell proliferation was determined by using a Cell Counting Kit-8 assay. (C) Migration and invasion of Eca109 cells following transfection with miR-NC, miR-455-5p mimics or miR-455-5p mimics + Rab31. ${ }^{*} \mathrm{P}<0.05$ vs. miR-NC group and ${ }^{\#} \mathrm{P}<0.05$ vs. miR-455-5p mimics group. (D) Migration and invasion of Eca109 cells following transfection with miR-NC, miR-455-5p inhibitor or miR-455-5p inhibitor + shR-Rab31. " $\mathrm{P}<0.05$ vs. miR-NC group and ${ }^{\circledR} \mathrm{P}<0.05$ vs. miR-455-5p inhibitor group. ESCC, esophageal squamous cell carcinoma; miR, microRNA; NC, negative control. 


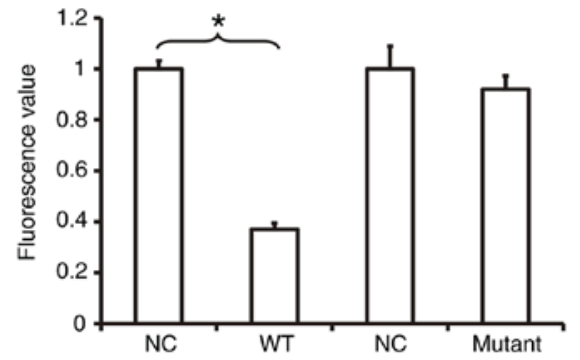

Rab31 $5^{\prime}$...AAUACUGUGGAUUAAUGGACUGU... miR-455-5p $3^{\prime}$ CACAUAUACGGGUACCUGACG

Mutant Rab31 5' ...AAUACUGUGGAUUAAGgAaGUAU...

miR-455-5p 3' CACAUAUACGGGUACCUGACG

Figure 6. The investigation between miR-455-5p and Rab31 mRNA was assessed using a dual luciferase reporter assay. Plasmids $(0.5 \mu \mathrm{g})$ with WT or mutant 3'-untranslated region DNA sequences were co-transfected into Eca109 cells with miR-455-5p mimics. After cultivation for $24 \mathrm{~h}$, ells were lysed using a dual luciferase reporter assay kit and the fluorescence intensity was measured using a GloMax 20/20 luminometer. Renilla was used as internal reference and the fluorescence values of each group of cells were measured. " $\mathrm{P}<0.05$ vs. $\mathrm{NC}$ group. miR, microRNA; ESCC, esophageal squamous cell carcinoma; $\mathrm{NC}$, negative control.

\section{Discussion}

The initiation and development of ESCC is a complex process that involves multiple genes, phases and steps. It is associated with gene mutation, abnormal expression, epigenetic inheritance and tumor stem cells $(31,32)$. As an important post-transcriptional regulator, miRNAs serve important roles in the occurrence and development of tumors (33). It has been reported that the miRNA expression profile is significantly altered in ESCC tissues and that various miRNA molecules act as oncogenes or tumor-suppressor genes in $\operatorname{ESCC}(34,35)$. In the present study it was revealed that miR-455-5p expression is downregulated in ESCC tissues and is negatively correlated with lymphatic metastasis and differentiation. In vitro experiments were used to demonstrate that miR-455-5p inhibits the proliferation, migration and invasion of ESCC cells. The results of bioinformatics and molecular biology studies indicate that miR-455-5p inhibits the proliferation, migration and invasion of ESCC cells by directly regulating the expression of Rab31. These results suggest that miR-455-5p downregulation promotes the occurrence and development of ESCC.

The miR-455 family includes two members, miR-455-5p and miR-455-3p, both of which participate in the proliferation, migration and invasion of several types of tumor cells (36). For example, miR-455-5p expression is downregulated in gastric cancer tissues and cells and miR-455-5p overexpression inhibits the proliferation and migration of gastric cancer cells by targeting Rab18, acting as a tumor-suppressor (23). In addition, miR-455-5p inhibits the proliferation and promotes the apoptosis of HCT116 colon cancer cells (20). The biological functions of miR-455 vary with tumor type and it may serve as an oncogene in some tumors. Li et al (21) reported that miR-455-3p promotes the proliferation and metastasis of triple-negative breast cancer by targeting EI24 gene expression. In oral squamous cell carcinoma, the TGF- $\beta /$ Smad signaling pathway upregulates miR-455-5p and promotes the proliferation, migration and invasion of tumor cells (24). The results of the present study demonstrate that miR-455-5p is significantly downregulated in ESCC tissues and is negative correlated with lymphatic metastasis and differentiation, suggesting that miR-455-5p may be an oncogene for ESCC. Transfection with miR-455-5p mimics inhibits the proliferation of Eca109 cells, whereas transfection with miR-455-5p inhibitor promotes proliferation. Transwell results revealed that the number of migrated and invading cells in the miR-455-5p mimics group was significantly lower compared with the $\mathrm{NC}$, whereas invasion and migration were increased in the miR-455-5p inhibitor group. This suggests that miR-455-5p inhibits the migration and invasion of ESCC cells.

miRNA molecules exert their biological functions mainly by inhibiting the expression of target genes. Bioinformatics used in the present study suggest that Rab31 is a potential target gene of miR-455-5p. Western blotting data revealed that Rab31 was downregulated in the miR-455-5p mimics group, whereas it is upregulated in the miR-455-5p inhibitor group, suggesting that miR-455-5p may exert its effect by regulating the expression of Rab31. Rab31 belongs to the Rab protein family and serves important regulatory roles in vesicle transport in cells (26). It has been reported that Rab31 is important for the apoptosis, proliferation and metastasis of tumors (26). For example, Rab31 inhibits apoptosis and promotes proliferation in hepatoma carcinoma cells by regulating the phosphoinositide 3-kinase/protein kinase B/B-cell lymphoma 2 (Bcl-2)/Bcl-2-associated X protein signaling pathway (27). Grismayer et al (37) reported that Rab31 is associated with the regulation of chemoresistance in breast cancer cells and affects the prognosis of patients (37). The results of the present study demonstrate that Rab31 overexpression in cells transfected with miR-455-5p mimics inhibits ESCC cell proliferation, while Rab31 downregulation in cells transfected with miR-455-5p inhibitor increases it. Transwell results revealed that Rab31 upregulation in the miR-455-5p mimics group facilitated the regulatory effect of miR-455-5p on ESCC cells, while Rab31 downregulation in the miR-455-5p inhibitor reduced the regulatory effect of miR-455-5p. Dual luciferase reporter assay results demonstrated that miR-455-5p directly binds with the 3'-UTR of Rab31 mRNA, suggesting that Rab31 is a direct target gene of miR-455-5p. However, the present study is not without limitations. The function of miR-455-5p in ESCC was not investigated in vivo and the mechanism by which Rab31 regulates ESCC development remains to be elucidated. In conclusion, the present study demonstrates that miR-455-5p inhibits the proliferation, migration and invasion of ESCC cells by directly regulating the expression of Rab31. miR-455-5p downregulation is an important factor that contributes to the occurrence and development of ESCC.

\section{Acknowledgements}

Not applicable.

\section{Funding}

No funding was received. 


\section{Availability of data and materials}

The datasets used and/or analyzed during the current study are available from the corresponding author on reasonable request.

\section{Authors' contributions}

YL and YT collaborated to design the study. YL and PL were responsible for experiments. YL and YT analyzed the data. All authors collaborated to interpret results and develop the manuscript. The final version of the manuscript has been read and approved by all authors, and each author believes that the manuscript represents honest work.

\section{Ethics approval and consent to participate}

All procedures performed in the current study were approved by the Ethics Committee of Tianjin Integrated Traditional Chinese and Western Medicine Hospital (Tianjin, China). Written informed consent was obtained from all patients or their families.

\section{Patient consent for publication}

Not applicable.

\section{Competing interests}

The authors declare that they have no competing interests.

\section{References}

1. Lin D, Ma L, Ye T, Pan Y, Shao L, Song Z, Jiang S, Chen H and Xiang J: Results of neoadjuvant therapy followed by esophagectomy for patients with locally advanced thoracic esophageal squamous cell carcinoma. J Thorac Dis 9: 318-326, 2017.

2. Mao Y, Han Y and Shi W: The expression of aplysia ras homolog I (ARHI) and its inhibitory effect on cell biological behavior in esophageal squamous cell carcinoma. Onco Targets Ther 10: $1217-1226,2017$

3. Cong $\mathrm{L}$ and $\mathrm{Hu} \mathrm{L}$ : The value of the combination of hemoglobin, albumin, lymphocyte and platelet in predicting platinum-based chemoradiotherapy response in male patients with esophageal squamous cell carcinoma. Int Immunopharmacol 46: 75-79, 2017.

4. He Z, Li G, Tang L and Li Y: SIX1 overexpression predicts poor prognosis and induces radioresistance through AKT signaling in esophageal squamous cell carcinoma. Onco Targets Ther 10 1071-1079, 2017.

5. Qiu B, Li J, Wang B, Wang Z, Liang Y, Cai P, Chen Z, Liu M, $\mathrm{Fu}$ J, Yang $\mathrm{H}$ and Liu H: Adjuvant therapy for a microscopically incomplete resection margin after an esophagectomy for esophageal squamous cell carcinoma. J Cancer 8: 249-257, 2017.

6. Morimoto H, Yano T, Yoda Y, Oono Y, Ikematsu H, Hayashi R Ohtsu A and Kaneko K: Clinical impact of surveillance for head and neck cancer in patients with esophageal squamous cell carcinoma. World J Gastroenterol 23: 1051-1058, 2017.

7. Zhao Y, Ma K, Yang S, Zhang X, Wang F, Zhang X, Liu H and Fan Q: MicroRNA-125a-5p enhances the sensitivity of esophageal squamous cell carcinoma cells to cisplatin by suppressing the activation of the STAT3 signaling pathway. Int J Oncol 53: 644-658, 2018

8. Sugase T, Takahashi T, Serada S, Nakatsuka R, Fujimoto M, Ohkawara T, Hara H, Nishigaki T, Tanaka K, Miyazaki Y, et al: Suppressor of cytokine signaling-1 gene therapy induces potent antitumor effect in patient-derived esophageal squamous cell carcinoma xenograft mice. Int J Cancer 140: 2608-2621, 2017.

9. Xue L, Nan J, Dong L, Zhang C, Li H, Na R, He H and Wang Y: Upregulated miR-483-5p expression as a prognostic biomarker for esophageal squamous cell carcinoma. Cancer Biomark 19: 193-197, 2017
10. Xie R, Wu SN, Gao CC, Yang XZ, Wang HG, Zhang JL, Yan W and Ma TH: MicroRNA-30d inhibits the migration and invasion of human esophageal squamous cell carcinoma cells via the post-transcriptional regulation of enhancer of zeste homolog 2 . Oncol Rep 37: 1682-1690, 2017.

11. Shi Q, Wang Y, Mu Y, Wang X and Fan Q: miR-433-3p inhibits proliferation and invasion of esophageal squamous cell carcinoma by targeting GRB2. Cell Physiol Biochem 46: 2187-2196, 2018.

12. Li SP, Su HX, Zhao D and Guan QL: Plasma miRNA-506 as a prognostic biomarker for esophageal squamous cell carcinoma. Med Sci Monit 22: 2195-2201, 2016.

13. Wang C, Li Q, Liu F, Chen X, Nesa EU, Guan S, Liu B, Han L, Tan B, Wang D, et al: Serum miR-1297: A promising diagnostic biomarker in esophageal squamous cell carcinoma. Biomarkers 21: 517-522, 2016.

14. Guan S, Wang C, Chen X, Liu B, Tan B, Liu F, Wang D, Han L, Wang L and Huang X, et al: miR-613: A novel diagnostic and prognostic biomarker for patients with esophageal squamous cell carcinoma. Tumour Biol 37: 4383-4391, 2016.

15. Xu H, Yao Y, Meng F, Qian X, Jiang X, Li X, Gao Z and Gao L: Predictive value of serum miR-10b, miR-29c, and miR-205 as promising biomarkers in esophageal squamous cell carcinoma screening. Medicine (Baltimore) 94: e1558, 2015.

16. Chen W, Chen L, Zhang Z, Meng F, Huang G, Sheng P, Zhang Z and Liao W: MicroRNA-455-3p modulates cartilage development and degeneration through modification of histone $\mathrm{H} 3$ acetylation. Biochim Biophys Acta 1863: 2881-2891, 2016.

17. Yao S, Tang B, Li G, Fan R and Cao F: miR-455 inhibits neuronal cell death by targeting TRAF3 in cerebral ischemic stroke. Neuropsychiatr Dis Treat 12: 3083-3092, 2016.

18. Wong N, Khwaja SS, Baker CM, Gay HA, Thorstad WL, Daly MD, Lewis JS Jr and Wang X: Prognostic microRNA signatures derived from the cancer genome atlas for head and neck squamous cell carcinomas. Cancer Med 5: 1619-1628, 2016.

19. Chai L, Kang XJ, Sun ZZ, Zeng MF, Yu SR, Ding Y, Liang JQ, Li TT and Zhao J: miR-497-5p, miR-195-5p and miR-455-3p function as tumor suppressors by targeting hTERT in melanoma A375 cells. Cancer Manag Res 10: 989-1003, 2018.

20. Zheng J, Lin Z, Zhang L and Chen H: MicroRNA-455-3p inhibits tumor cell proliferation and induces apoptosis in HCT116 human colon cancer cells. Med Sci Monit 22: 4431-4437, 2016.

21. Li Z, Meng Q, Pan A, Wu X, Cui J, Wang Y and Li L: MicroRNA-455-3p promotes invasion and migration in triple negative breast cancer by targeting tumor suppressor EI24. Oncotarget 8: 19455-19466, 2017.

22. Chai J, Guo D, Ma W, Han D, Dong W, Guo H and Zhang Y: A feedback loop consisting of RUNX2/LncRNA-PVT1/miR-455 is involved in the progression of colorectal cancer. Am J Cancer Res 8: 538-550, 2018.

23. Liu J, Zhang J, Li Y, Wang L, Sui B and Dai D: miR-455-5p acts as a novel tumor suppressor in gastric cancer by down-regulating RAB18. Gene 592: 308-315, 2016.

24. Cheng CM, Shiah SG, Huang CC, Hsiao JR and Chang JY: Up-regulation of miR-455-5p by the TGF- $\beta$-SMAD signalling axis promotes the proliferation of oral squamous cancer cells by targeting UBE2B. J Pathol 240: 38-49, 2016.

25. Kotzsch M, Kirchner T, Soelch S, Schäfer S, Friedrich K, Baretton G, Magdolen V and Luther T: Inverse association of rab31 and mucin-1 (CA15-3) antigen levels in estrogen receptor-positive $(\mathrm{ER}+)$ breast cancer tissues with clinicopathological parameters and patients' prognosis. Am J Cancer Res 7: 1959-1970, 2017.

26. Pan Y, Zhang Y, Chen L, Liu Y, Feng Y and Yan J: The critical role of Rab31 in cell proliferation and apoptosis in cancer progression. Mol Neurobiol 53: 4431-4437, 2016.

27. Sui Y, Zheng X and Zhao D: Rab31 promoted hepatocellular carcinoma (HCC) progression via inhibition of cell apoptosis induced by PI3K/AKT/Bcl-2/BAX pathway. Tumour Biol 36: 8661-8670, 2015.

28. Wang J, Wu N, Zheng QF, Yan S, Lv C, Li SL and Yang Y: Evaluation of the 7th edition of the TNM classification in patients with resected esophageal squamous cell carcinoma. World J Gastroentero 20: 18397-18403, 2014.

29. Ke W, Zeng L, Hu Y, Chen S, Tian M and Hu Q: Detection of early-stage extrahepatic cholangiocarcinoma in patients with biliary strictures by soluble B7-H4 in the bile. Am J Cancer Res 8: 699-707, 2018. 
30. Livak KJ and Schmittgen TD: Analysis of relative gene expression data using real-time quantitative PCR and the 2(-Delta Delta C(T)) method. Methods 25: 402-408, 2001.

31. Zhang W, Hong R, Xue L, Ou Y, Liu X, Zhao Z, Xiao W, Dong D, Dong L and Fu M: Piccolo mediates EGFR signaling and acts as a prognostic biomarker in esophageal squamous cell carcinoma. Oncogene 36: 3890-3902, 2017.

32. Mei LL, Qiu YT,Zhang B and Shi ZZ: MicroRNAs in esophageal squamous cell carcinoma: Potential biomarkers and therapeutic targets. Cancer Biomark 19: 1-9, 2017.

33. Meng X, Chen X, Lu P, Ma W, Yue D, Song L and Fan Q: miR-202 promotes cell apoptosis in esophageal squamous cell carcinoma by targeting HSF2. Oncol Res 25: 215-223, 2017.

34. Yi J, Jin L, Chen J, Feng B, He Z, Chen L and Song H: miR-375 suppresses invasion and metastasis by direct targeting of SHOX2 in esophageal squamous cell carcinoma. Acta Biochim Biophys Sin (Shanghai) 49: 159-169, 2017.
35. Ren Y, Chen Y, Liang X, Lu Y, Pan W and Yang M: miRNA-638 promotes autophagy and malignant phenotypes of cancer cells via directly suppressing DACT3. Cancer Lett 390: 126-136, 2017.

36. Wang J, Wang Y, Sun D, Bu J, Ren F, Liu B, Zhang S, Xu Z, Pang S and Xu S: miR-455-5p promotes cell growth and invasion by targeting SOCO3 in non-small cell lung cancer. Oncotarget 8: 114956-114965, 2017.

37. Grismayer B, Sölch S, Seubert B, Kirchner T, Schäfer S, Baretton G, Schmitt M, Luther T, Krüger A, Kotzsch M and Magdolen V: Rab31 expression levels modulate tumor-relevant characteristics of breast cancer cells. Mol Cancer 11: 62, 2012.

(i) (-) This work is licensed under a Creative Commons Attribution-NonCommercial-NoDerivatives 4.0 International (CC BY-NC-ND 4.0) License. 